\title{
Performance Evaluation of Underwater Wireless Sensor Networks with OPNET
}

\author{
Jesús Llor, Manuel P. Malumbres, Pablo Garrido \\ Dept. of Physics and Computer Engineering \\ Universidad Miguel Hernández \\ Avenida de la Universidad S/N, Edificio Alcudia \\ 03202, Elche (Alicante) Spain \\ +34966658393 \\ illor@umh.es, mels@umh.es, pgarrido@umh.es
}

\begin{abstract}
One of the main difficulties when modeling Underwater Wireless Sensor Network (UWSN) has to do with the environment characteristics and the acoustic signal typically used. As stated in other works, the accuracy of the acoustic propagation model and the network scenario conditions are critical to obtain reliable results. In this work we proposed a simulator framework for UWSN modeling. For that purpose we have considered the information provided by global databases (temperature, salinity, etc.) located within the network. Namely, the bathymetry and floor sediment, node depth, wave effect, and other factors may affect to the underwater signal propagation behavior. The propagation model is calculated using Bellhop ray tracing tool in order to get the closest representation to the real behavior of the acoustic signal propagation. All these tools are integrated in OPNET Modeler. Finally, we have run several experiments in different locations testing network performance with a simple MAC protocol.
\end{abstract}

\section{Categories and Subject Descriptors}

C.2.1 [Network Architecture and Design];

I.6.3 [Simulation and Modeling]: Simulation applications;

I.6.5 [Simulation and Modeling]: Model Development.

\section{General Terms}

Algorithms, Measurement, Performance, Design.

\section{Keywords}

Underwater acoustics, Acoustic channel model, Wireless sensor networks, Network simulation, OPNET, MATLAB.

\section{INTRODUCTION}

Terrestrial Wireless Sensor Networks (TWSN) have been developed in the last two decades. As a result of this research the physical layer has been modeled and a wide range of MAC protocols, routing protocols and applications have been worked out. But in order to make this possible, standardization and simulation tools are needed by the researchers and commercial companies to test and validate the algorithms before implementing them in real hardware prototypes.

Permission to make digital or hard copies of all or part of this work for personal or classroom use is granted without fee provided that copies are not made or distributed for profit or commercial advantage and that copies bear this notice and the full citation on the first page. To copy otherwise, to republish, to post on servers or to redistribute to lists, requires prior specific permission and/or a fee.

SIMUTOOLS 2011, March 21-25, Barcelona, Spain

Copyright $\odot 2011$ ICST 978-1-936968-00-8

DOI 10.4108/icst.simutools.2011.238581
The most outstanding simulator tools used in terrestrial networks are Network Simulator 2 (ns2) [14] and OPNET [9]. In those simulators you can find protocol models that are currently used by mobile wireless networks as 802.11, UMTS/3G, Zigbee, etc. Underwater Wireless Sensor Networks (UWSN) are just arising in the world of simulators, so a lot of simulation tools are currently available. Most of them partially cover some aspects of the network architecture, being difficult to find a complete and reliable UWSN simulation tool.

Many of these simulators are based on terrestrial wireless network models reusing many elements from them, other simulators are dedicated to model underwater acoustic propagation and are available in different programming languages like python, fortran $90, \mathrm{c} / \mathrm{c}++$, etc. In this work we will gather several simulation proposals, analyzing their benefits and drawbacks in order to build a new complete and reliable simulation framework, that allows the design and performance evaluation of new underwater network protocols under a friendly user interface.

In particular, we are especially motivated to define detailed models about the underwater environment (temperature, bathymetry, surface activity, etc.) and the acoustic signal behavior because the simulation results of particular network architecture should be as close as possible to the results that would be obtained in a real scenario under similar conditions. This is a great challenge, since the acoustic signal modeling in underwater scenarios is very complex and depends on many external factors like temperature, floor sediment composition, wave activity, environmental noise, etc. So, when evaluating MAC, routing or higher level protocols in a UWSN, the fact of using different underwater signal propagation models may seriously conditioning the final simulation results.

The rest of paper is organized as follows: Section 2 gives an overview of related work introducing several simulator tools. Section 3 stands an overview of the simulator performance describing in detail the steps that have been followed to make an integrated simulation tool. Section 4 shows some simulation tests to validate the proposed tool. Section 5 presents analysis of some of the simulation results, and finally, in Section 6, conclusions and future works are commented. 


\section{RELATED WORK}

Among the simulation tools suitable for UWSN, we have chosen three of the most outstanding ones, with different approach levels and source code available.

AUVNetSim [4] is an example of a new simulator proposal, here the author uses python language to build an underwater wireless network scenario. Highlights in this simulator are the availability of the DACAP [10] MAC protocol and the Focus Beam Routing (FBR) [5] algorithm that includes transmission power control. However, the physical layer is too simple, and so different environment conditions cannot be properly modeled.

Another simulator proposed by Xie and Gibson [16] defines a complex underwater acoustic propagation model based on the Monterrey Miami Parabolic Equation (MMPE), which it is implemented in OPNET Modeler and tested with popular MAC protocols like Aloha and CSMA. This simulator stands out because it describes the physical layer at high level of details, being possible to define a scenario considering more environmental parameters like the effect of the waves and node depth. The main drawback of this model is that before doing network simulations, several propagation model parameters need to be computed, being this process high time-consuming. Also, the model only works on static scenarios with static conditions across the simulation period.

The World Ocean Simulator System (WOSS) [2][3] seems to be one of latest and most complete simulator tools at the moment. It is implemented in the ns2 simulator. It uses world databases that measure the Sound Speed Profile (SSP), bathymetry and floor sediment composition such as the General Bathymetric Chart of the Oceans (GEBCO) and National Oceanic and Atmospheric Administration (NOAA). Combining this data with the scenario information like the latitude, longitude and depth position of the nodes, it creates environmental files that describe the properties of the scenario. In this work, authors have validated Bellhop propagation model with real experimental data obtained in waters surrounding Pianosa Island (Italy), showing that Bellhop model results closely resemble the ones measured in a real underwater scenario.

\section{SIMULATOR PROPOSAL}

Based on our previous work on OPNET [9] we will develop a propagation model suitable for acoustic networks which capture the best of the currently available proposals. The main goal is to create a simulator framework able to work with scenarios located anywhere in the world. So, the obtained results will be in accordance to the local environmental conditions and will be closer to the ones measured in the real world.

Our purpose is to pick the world information from global databases and combine it with the OPNET scenario design to produce a set of environmental files. With this information, OPNET Modeler connects to MATLAB [7] through its interface calling to the Bellhop ray tracing tool [11] (propagation model), giving as a result several files including information such as amplitude, travel times, ray coordinates and the acoustic pressure map. With these files OPNET is able to perform the simulation of the acoustic signal propagation and determine the set of potential destination nodes that will be able to receive the signal sent by a single source node.

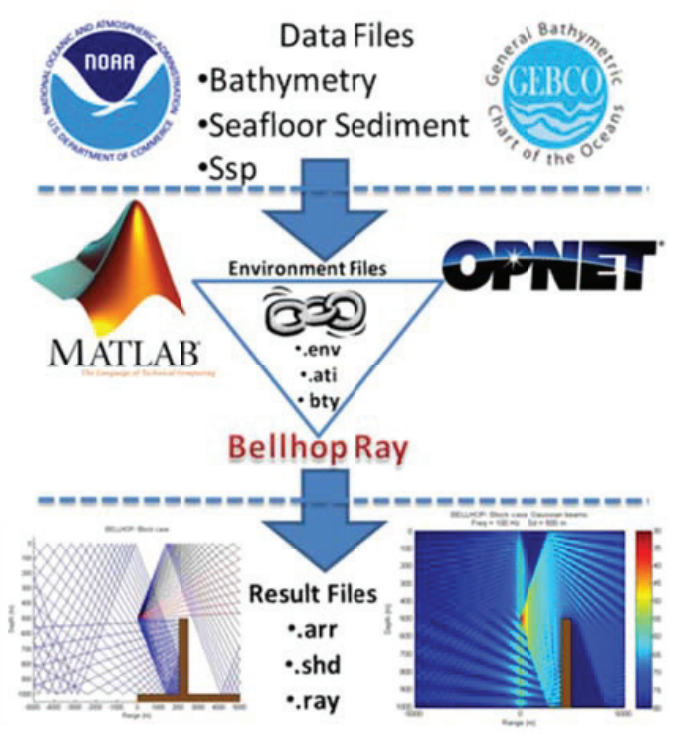

Figure 1. Simulator Summary

In Figure 1, we show the three steps required to complete the process: (a) obtaining data from global databases, (b) creating the environment files, and (c) executing the Bellhop ray tracing tool to get the results for the propagation model.

As a result of this process, the signal delay and attenuation between every pair of nodes is known, allowing to OPNET to start with the network simulation. This process is described in detail in the next sections.

\subsection{World Databases}

Three main world databases will be used to model the environment files and define in detail the characteristics of the scenario where the network is located.

- Bathymetry [1]: Is provided by the GEBCO. A file containing the world bathymetry data in a global 30 arcsecond grid released in January 2009 and updated in November 2009. This information will be used either for the bottom scenario relief as well as for the sound speed profile generation.

- Seafloor Sediment [8]: The National Geophysical Data Center (NGDC) from the NOAA provides a "Deck41" database that contains surficial sediment descriptions for over 36,000 seafloor samples worldwide. Mainly we have ten types of floors in the ocean: gravel, sand, silt, clay, ooze, mud, rocks, organic, nodules and hard-bottom. If no one is available no-data value will be return.

- $\quad$ Sound Speed Profile (SSP) [15]: Provided by the World Ocean Atlas (WOA) of NOAA. Contains information of the evolution of worldwide sound speed along the year. It can be specified and averaged over the year, the season or, even more detailed, by month. In Figure 2, we can see average values of sound speed depending on the latitude and the season of the year. It can be appreciated that the greatest differences are found in shallow waters. 


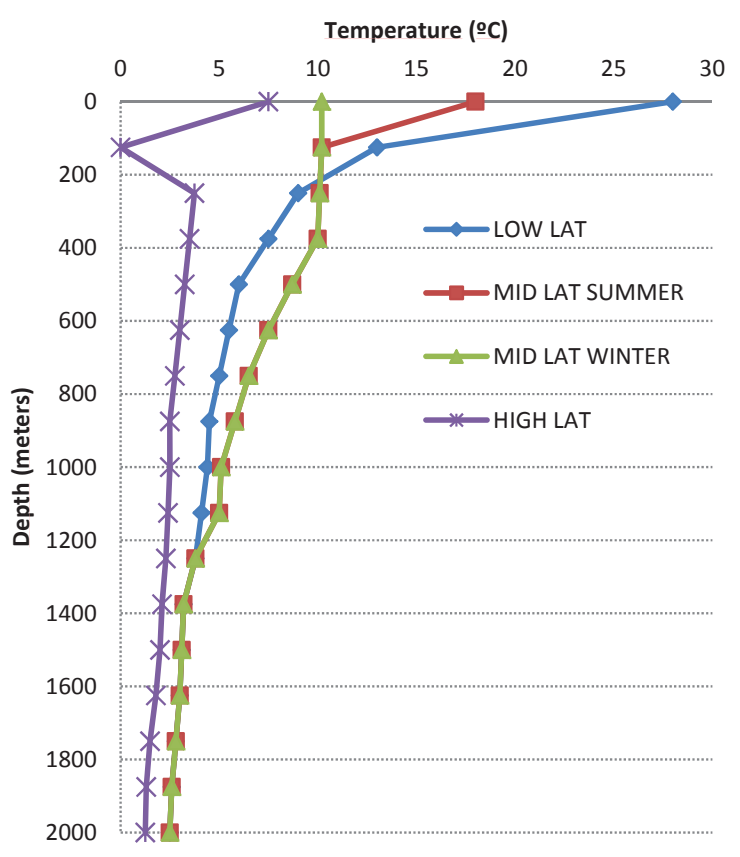

Figure 2. Average Temperature Values

All these databases are formatted in Network Common Data Form (netCDF); we access to them from MATLAB through the mexnc library and the Snctools [13]. In addition, MATLAB is invoked from OPNET by using the MATLAB interface for executing " $\mathrm{C}$ " code. A full explanation of the configuration can be found in [12].

\subsection{Environmental Files}

Once the data are available from OPNET, they are combined together with the information of the nodes (latitude, longitude and depth) and global parameters of the scenario such as frequency, wave information (height and length), the month of the year, etc. The results are three environment files required by Bellhop [11]:

- $\quad$ Environment file (*.env): The general structure of this file is shown in Figure 3. The values of the Sound Speed Block and the Bottom Block are gathered from the databases. The Array block is data from OPNET node global position, and the Surface line, Output Block and Beam block are simulation global parameters.

- Bathymetry file (*.bty): Consists of a two columns table including range $(\mathrm{km})$ and depth $(\mathrm{m})$. This file is created from the databases bases taking into account the global position of the network nodes.

- $\quad$ Altimetry file (*.ati): Compounds of a two columns: namely range $(\mathrm{km})$ and depth $(\mathrm{m})$. This file is created with two global parameters "Wave Height" and "Wave Length".

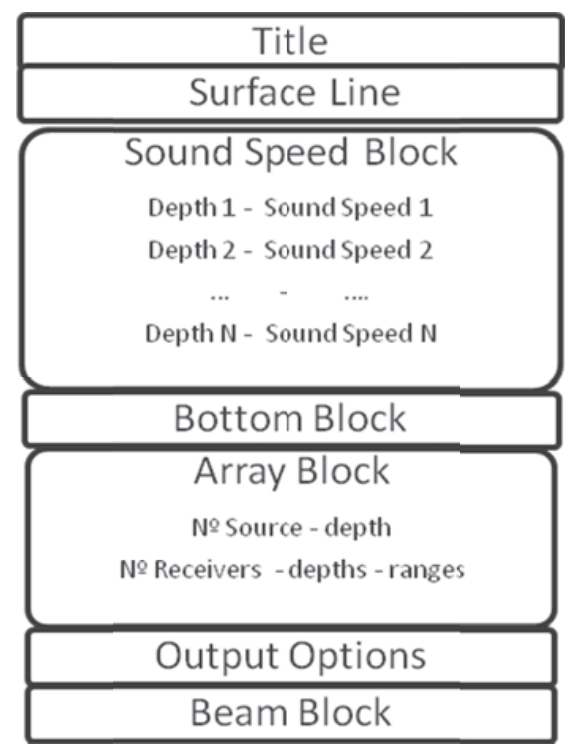

Figure 3. Environment file definition

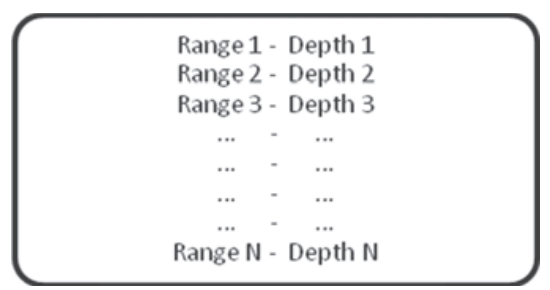

Figure 4. Bathymetry and Altimetry file definition

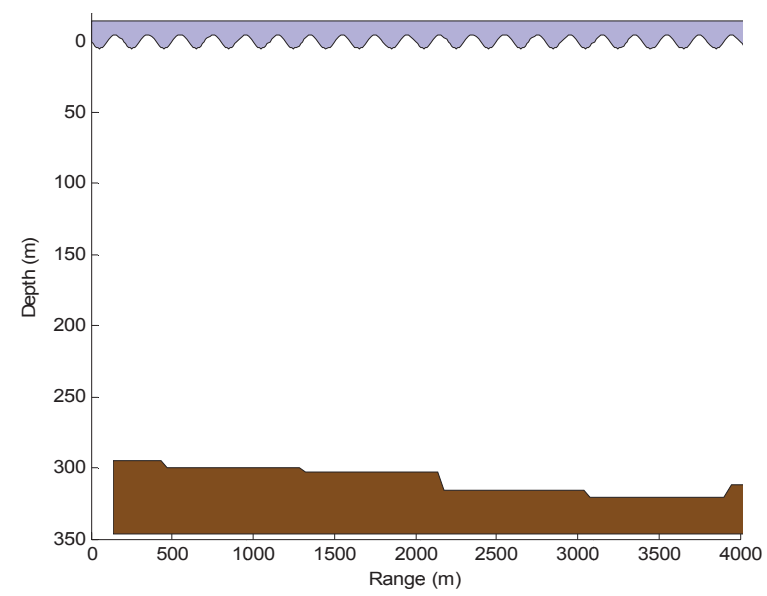

Figure 5. Plotting Bathymetry and Altimetry file data 
Based on data from bathymetry and altimetry files we can define the surface or wave shape and the bottom relief, as shown in the upper part of Figure 5, a 2D vertical slice of underwater scenario, where the network is placed. So, in this way we can define a simulation scenario placed in any part of the world with real data about the local underwater environment characteristics.

\subsection{Bellhop Ray Tracing Tool}

Again, we use the MATLAB interface from OPNET to communicate and execute the Bellhop ray tracing tool taking the files created in the previous step as input. Depending on the desired output option, different result files will be created:

- Option A: a *arr file is created with the information of the amplitude and travel times of the rays that arrive at the receiver position.

- Option R: a *.ray file is conformed to the coordinates of every ray traced. So, we are able to build ray trajectories with all the distortions and reflections caused by their propagation along the scenario. They will be very different depending on the proximity to the surface and the height and length of the waves, as well as the proximity and shape of the bottom. In addition, the types of sediment that can be found in the bottom may affect the ray propagation.

- $\quad$ Option C: a *.shd file is generated with the acoustic pressure found in every point of a grid that cover the entire scenario. Acoustic pressure may be calculated in a coherent, incoherent or semi-coherent way.

\section{SIMULATION SCENARIOS}

In order to testing the simulator, the same node deployment network has been placed in several world locations (shown at Figure 6). The depths of network nodes will vary depending on the scenario we simulate, being different if the network is located in shallow or deep waters.

Next, we show the results for three different locations. In each of them, the environmental conditions differ for the bathymetry, the sound speed profile, the sediment floor, and the altimetry which is based on wave parameters.

The main aim when choosing these locations was to test very different scenarios with different environment conditions (wave activity, depths, etc.). So, we can evaluate the performance of the same network at different locations measuring the impact of the scenario parameters in the simulation results. The extension of the network will be a square of $4000 \times 4000$ meters for all the location with different depths.

\subsection{Valencia - Spain}

The first place is located in coordinates $39^{\circ} 48^{\prime} 13.14 " \mathrm{~N}$ and $0^{\circ} 4^{\prime} 34.53$ "O. The node depth varies from 5 to 20 meters, the wave height is fixed to 0.5 meters and the wave length is 80 meters. This is an example of shallow waters with a low altimetry shape. The sediment floor of the bottom in this scenario is gravel. We can see the node deployment in Google Earth in Figure 6(a) and the results for the Bellhop ray execution are shown in Figures 7 and 8 .

This example could represent a typical network scenario close to the coast. The bottom relief in the scenario is deeper as it goes farther from the coast. Results show how the rays reflect a great number of times in the bottom and the surface.

\subsection{Hawaii - USA}

The second scenario is placed in coordinates $20^{\circ} 39^{\prime} 13.10^{\prime \prime} \mathrm{N}$ and $156^{\circ} 44^{\prime} 39.84^{\prime \prime O}$. The node depth varies from 10 to 300 meters and the wave height is fixed to 8 meters with a length of 150 meters. This is an example of non-shallow water with a large ocean waves specified in the altimetry file. The sediment floor in this region is ooze. The node deployment in Google Earth is shown in Figure 6(b) and the results of Bellhop ray execution are depicted in Figures 9 and 10.

This scenario represents those network deployments within the surroundings of a group of islands.

\subsection{Random Location (Atlantic Ocean)}

Finally, our last scenario is located at a random place in the middle of Atlantic Ocean, to test a deep water case. The GPS coordinates are $4^{\circ} 52^{\prime} 4.80 \prime \mathrm{N}$ and $34^{\circ} 57^{\prime} 0.00^{\prime \prime} \mathrm{O}$. The node depth varies between 50 and 1000 meters.

The surface activity is defined with waves of 10 meters height and 100 meters length. The sediment floor in this region is mud and organic. We can see the node deployment in Google Earth in Figure 6(c) and the result for the Bellhop ray execution in Figures 11 and 12 .

In this scenario, the source and destination nodes are far from the bottom and the waves, so both do not have a great effect in the way the rays behave. This scenario tries to show a network in the middle of an ocean, far away from the coast and in deep waters areas.

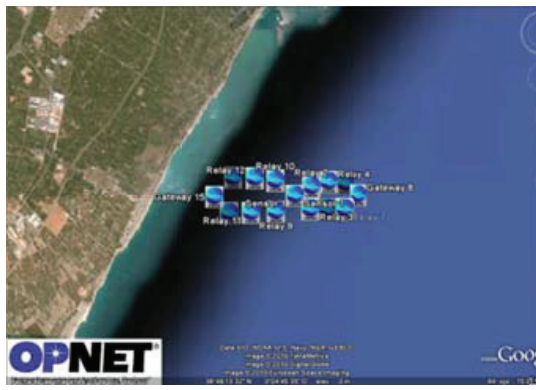

(a) Valencia

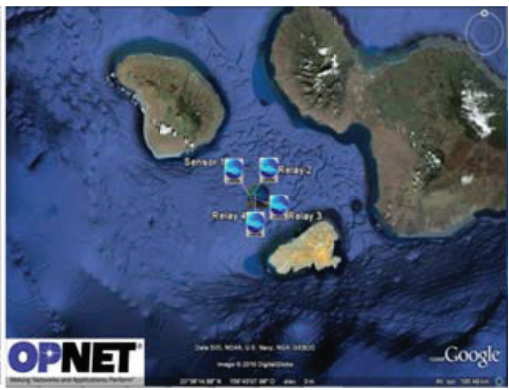

(b) Hawaii

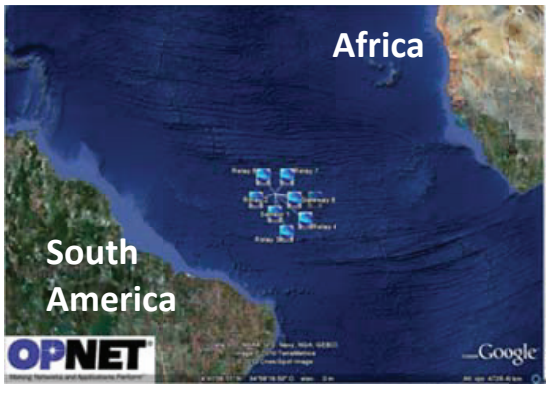

(c) Random location in the middle of Atlantic Ocean

Figure 6. The same network topology deployed 


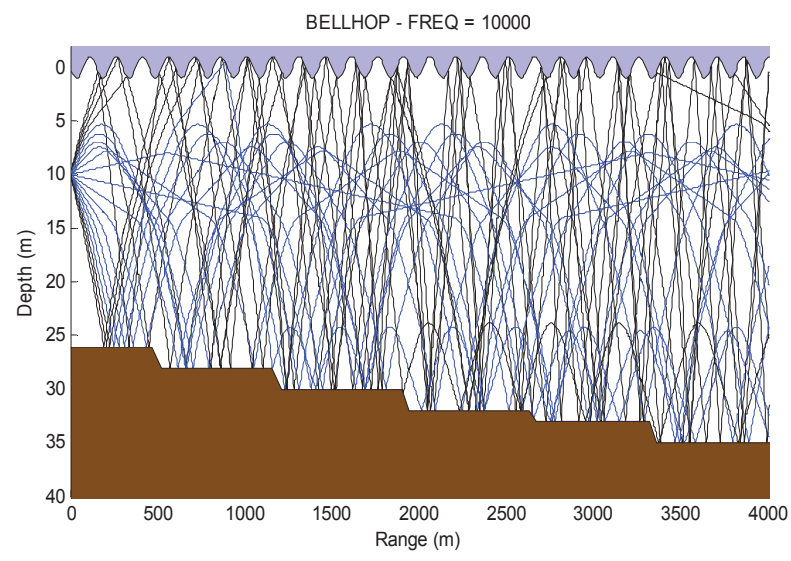

Figure 7. Bellhop Ray Map for Valencia

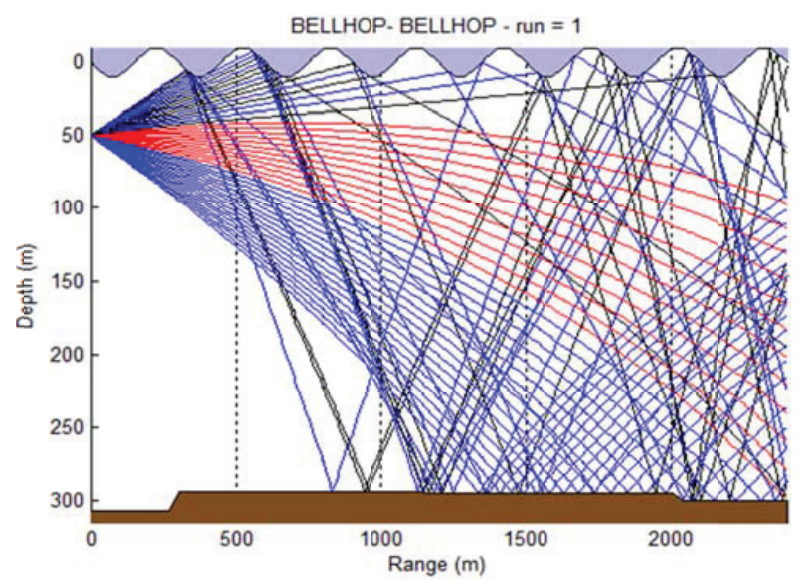

Figure 9. Bellhop Ray Map for Hawaii

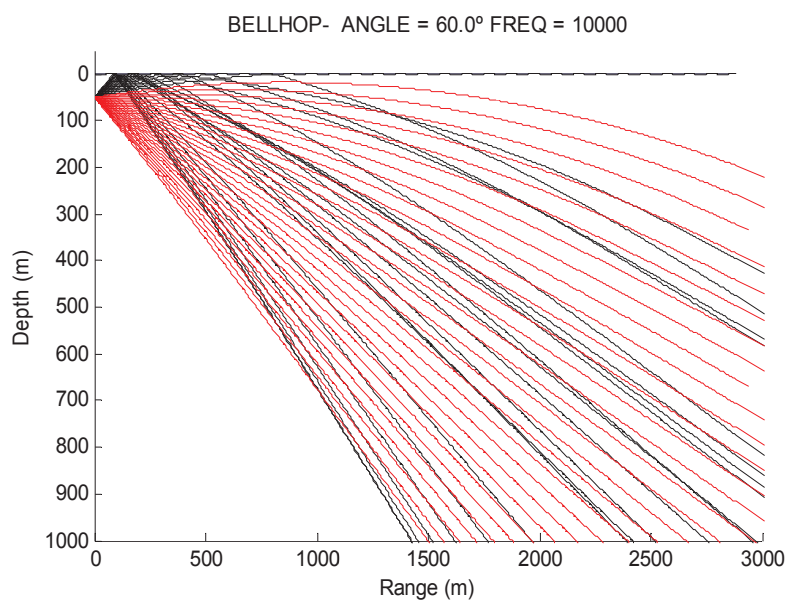

Figure 11. Bellhop Ray Map for Atlantic Ocean (Random Location)

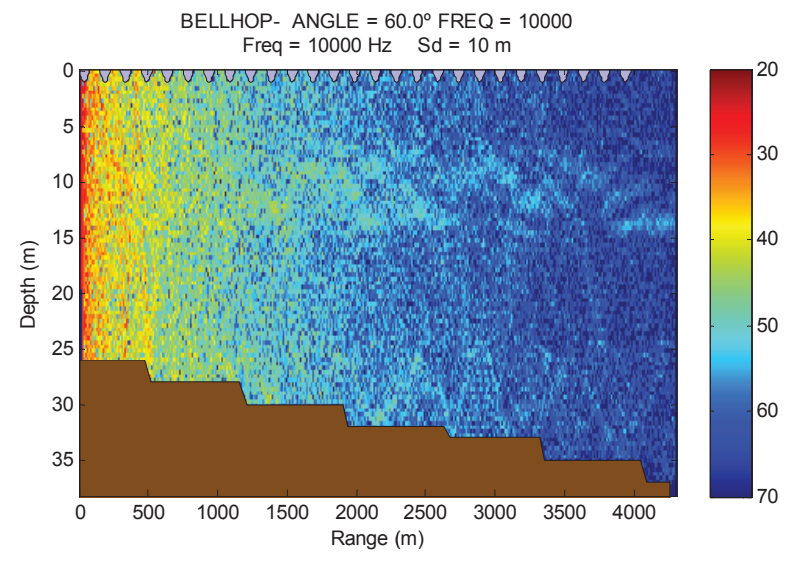

Figure 8. Bellhop Acoustic Pressure Map for Valencia

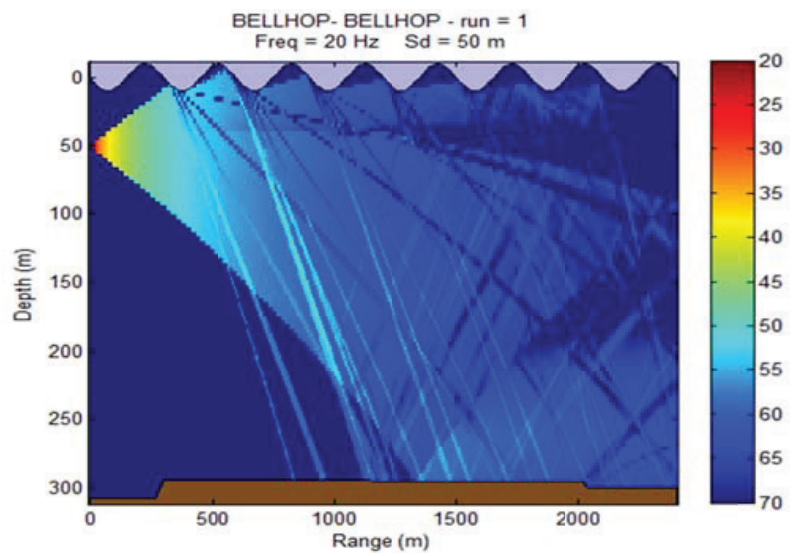

Figure 10. Bellhop Acoustic Pressure Map for Hawaii

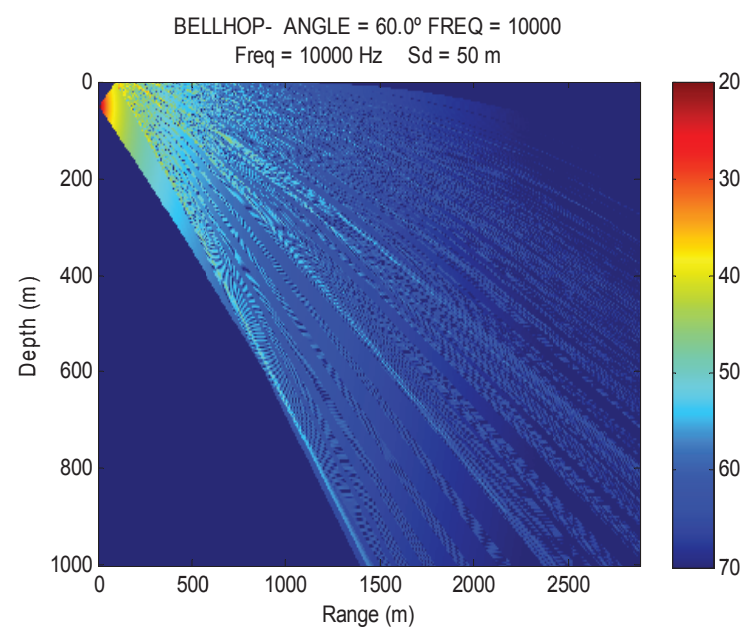

Figure 12. Bellhop Acoustic Pressure Map for Atlantic Ocean (Random Location) 


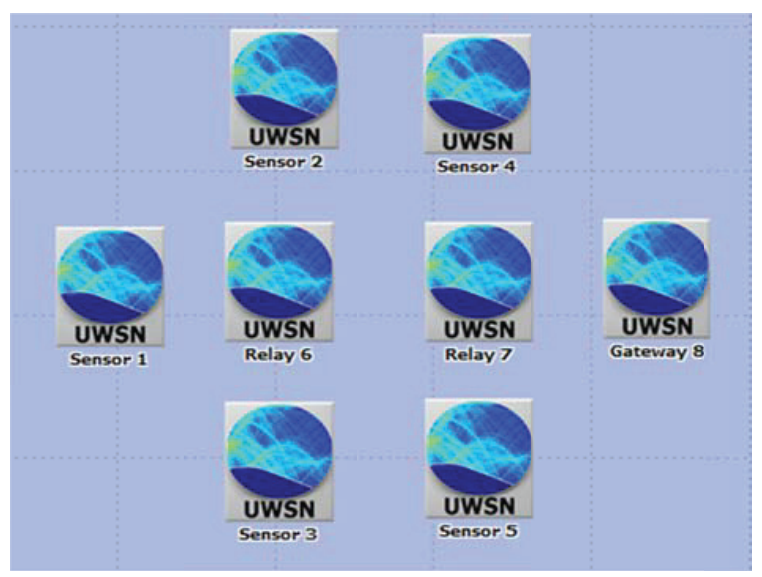

Figure 13. Network deployment

\section{SIMULATION TESTS}

The same simple network deployment (figure 13) is placed in the different scenarios chosen for the previous simulation test, five sensor nodes generates the network traffic load, two relay nodes only forward packets and there is one sink node that receives the information from the sensors. The distances between nodes vary from 1300 to 1500 meters

The purpose of the simulation tests is to run a simple MAC protocol such as ALOHA in all the different scenarios with different conditions in order to analyze the behavior of the simulation tool. This will lead us to the conclusion of how important is to have a realistic simulator to test new protocols and validate them under multiple conditions in which it can be involved.

The simulation duration is 3 hours which last between 4 and 6 minutes in an Intel ${ }^{\circledR}$ Core ${ }^{\mathrm{TM}} 2$ Duo T8100 $2.10 \mathrm{GHz}$ with $3 \mathrm{~GB}$ of RAM memory. The main performance metrics we will show are network collisions and gateway throughput. The temperature values are an annual average for each zone. The most significant parameters used for the simulations appear in Table 1:

Table 1. Simulation Parameters

\begin{tabular}{|c|c|c|c|}
\hline Parameters & Valencia & Hawaii & Atlantic \\
\hline Wave Height (meters) & 0.5 & 2 & 6 \\
\hline Wave Length (meters) & 25 & 100 & 150 \\
\hline Node Depth (meters) & 15 & 50 & 50 \\
\hline Scenario Depth (depth) & 26 & 300 & 5000 \\
\hline Seafloor Sediment & Gravel & Ooze & $\begin{array}{c}\text { Mud } / \\
\text { Organic }\end{array}$ \\
\hline Frequency (Khz) & & 10 \\
\hline Pkt Interarrival Time (s) & \multicolumn{3}{|c|}{5 to1000 } \\
\hline
\end{tabular}

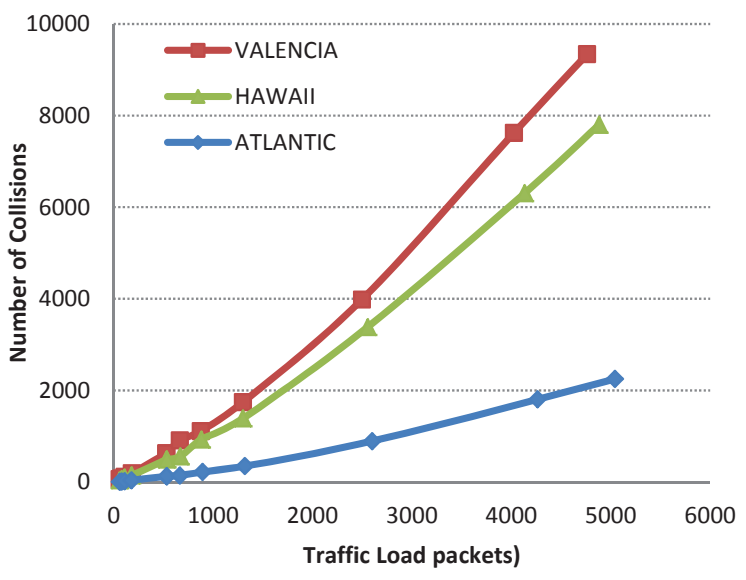

Figure 14.Collisions in different scenarios

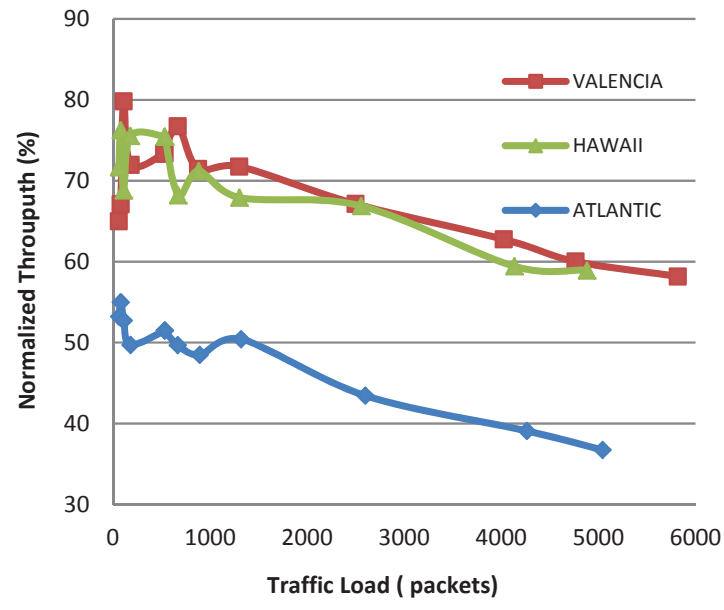

Figure 15.Collisions in different scenarios

All the simulation results were obtained by averaging 15 simulation runs per traffic load, resulting in a total of 300 simulations per scenario.

As expected, even though using the same network deployment the simulation results will differ from one scenario to another, as can be seen from figures 14 and 15. Apart from using different locations, the protocol has been tested under different conditions (e.g. different wave activity for each scenario).

In the first case (Valencia) with a low wave shape scenario, in each transmission the nodes more neighbors are reached, which means that less collisions occurs than in the other locations as more packets are lost due to the wave effect among other facts like the temperature difference, the bottom reflection, etc. We also evaluate the normalized throughput in the gateway (packets received in gateway node divided by all packets generated in the five sensors). 
As a result of using Aloha protocol, the normalized throughput falls as the generation of packets increases. It is appreciable that the scenario with less collision is also the one with less throughput, this happens because the acoustic signal of sensor nodes reach to less neighbors and despite generating the same number of packets there fewer packets travelling in the medium and thus less overall throughput. For instance, during a single simulation the average number of reachable neighbors for Relay 6 in Valencia scenario is around four, in Hawaii between four and three and in Atlantic around two. Nevertheless the important point from these graphs is not the behavior of the MAC protocol but the difference of using the same MAC protocol with the same network parameters in different environment conditions and though having different results.

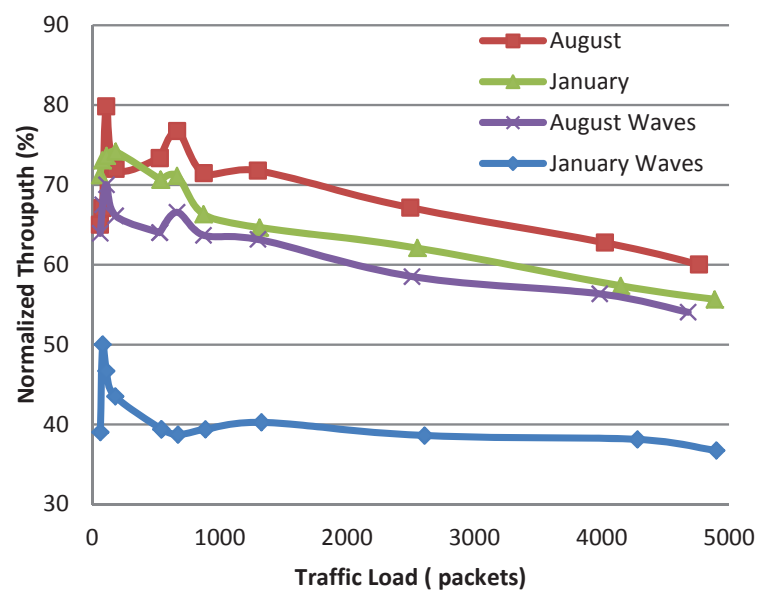

Figure 16.Normalized Throughput in Valencia (Season \& Waves)

Now to emphasize the environment effects we choose one single scenario and run different simulations with changing conditions. We have done simulations for every month of the year and with multiple wave shapes (12 months and 5 ocean wave shapes and 15 traffic loads). In the graph shown in figure 16 we show only two months (January and August) and each one with and without wave effect to highlight both characteristics and their influence. The shape of the ocean waves in the scenarios that include this effect are 2 meters height and 80 meters length.

From figure 16 we can draw that there is a small throughput difference between the simulations of January and August without the wave effect. There is also a slightly higher variation in the results when comparing the normal August scenario against the same month with waves, but the waves in winter season have a considerable impact on overall network throughput, reducing it up to $50 \%$. So, we can conclude that in this particular case of shallow water scenario where the temperature variation is greater than in deeper scenarios (see Figure 2), the effect of the season is appreciable and its combination with the ocean waves can change completely the network performance.

\section{CONCLUSIONS}

We have presented a powerful simulator tool to model underwater wireless sensor network scenarios all around the world. It is composed by well-known tools like OPNET network simulator, MATLAB and the Bellhop ray tracing tool. In addition, ocean databases were used to proper modeling the environmental scenario characteristics of a specific world location. The results of Bellhop propagation model has been tested and validated in real scenarios and the code is freely available as well as the protocols and databases used in this proposal. Simply using the simulator to place the network it will automatically generate the whole scenario, extracting from the databases the necessary information to build the environment conditions as well as the wave pattern. The interface can be used by non-developer users, being ease of use and fully configurable; this is an advantage over other proposals where in order to run a simulation there is a need to introduce the simulator and its implementation to the users.

Although the simulator is enough accurate with the acoustic signal behavior, it is necessary to achieve a trade-off between accuracy and simulation complexity in order to find out the desired balance. This is one of the mayor challenges for future work and next simulator improvements. Reducing the simulation times will let us perform large scenario simulations in reasonable times and, increasing the simulator scalability.

As previously remarked, the importance of testing new protocols proposals not only in ideal conditions but also when the physical parameters have a great impact on the performance is an essential point to validate our proposals in scenarios very close to real ones.

\section{ACKNOWLEDGMENTS}

This work was supported by the Ministry of Science and Education of Spain under grant DPI2007-66796-C03-03.

\section{REFERENCES}

[1] General bathymetric chart of the oceans. [Online]. Available $=$ http://www.gebco.net

[2] Guerra, F. 2009. A performance comparison of MAC protocols for underwater networks using a realistic channel simulator, OCEANS 2009, MTS/IEEE Biloxi - Marine Technology for Our Future: Global and Local Challenges, vol., no., pp.1-8, 26-29 Oct. 2009. DOI= http://ieeexplore.ieee.org/stamp/stamp.jsp?tp=\&arnumber= 5422249\&isnumber $=5422059$

[3] Guerra, F. 2009. World ocean simulation system (WOSS): a simulation tool for underwater networks with realistic propagation modeling. In Proceedings of the Fourth ACM International Workshop on UnderWater Networks (WUWNet ). ACM, New York, NY, USA. DOI=http://doi.acm.org/10.1145/1654130.1654134

[4] Jornet, J.M. 2008. AUVNetSim: A Simulator for Underwater Acoustic Networks. [Online]. Available=http://sourceforge.net/projects/auvnetsim/. 
[5] Jornet, J.M., Stojanovic, 2008. M. Focused beam routing protocol for underwater acoustic networks. In Proceedings of the third ACM international workshop on Underwater Networks (WuWNeT '08). ACM, New York, NY, USA, 75-82. DOI=http://doi.acm.org/10.1145/1410107.1410121

[6] Llor, J. 2009. Analyzing the behavior of acoustic link models in underwater wireless sensor networks. In Proceedings of the 4th ACM workshop on Performance monitoring and measurement of heterogeneous wireless and wired networks (PM2HW2N '09). ACM, New York, NY, USA, 9-16. DOI= http://doi.acm.org/10.1145/1641913.1641915

[7] MATLAB R2009a [Online]. Available= http://www.mathworks.com/

[8] National geophysical data center, seafloor surficial sediment descriptions. [Online]. Available $=$ http://www.ngdc.noaa.gov/mgg/geology/deck 41.html

[9] OPNET Modeler v15.0 Reference Manual OPNET Technologies Inc. OPNET Modeler [Online]. Available $=$ http://www.opnet.com/

[10] Peleato, B., Stojanovic, 2007. Distance aware collision avoidance protocol for ad hoc underwater acoustic sensor networks, IEEE Commun. Lett., vol. 11, no. 12, pp. 10251027.
[11] Porter, M. Bellhop code. [Online]. Available: http://oalib.hlsresearch.com/Rays/index.html

[12] Seppälä, J.M., 2009. Simulating devices with adaptive antenna arrays using OPNET integrated with MATLAB. In Proceedings of the 4th ACM workshop on Performance monitoring and measurement of heterogeneous wireless and wired networks (PM2HW2N '09). ACM, New York, NY, USA, 159-166. DOI= http://doi.acm.org/10.1145/1641913.1641936

[13] Snctools for MATLAB [Online]. Available= http://mexcdf.sourceforge.net/.

[14] The Network Simulator [Online]. Available= http://www.isi.edu/nsnam/ns/

[15] World ocean atlas. [Online]. Available $=$ http://www.nodc.noaa.gov/OC5/WOA05/pr_woa05.html

[16] Xie, G., Gibson, J., 2006. Incorporating Realistic Acoustic Propagation Models in Simulation of Underwater Acoustic Networks: A Statistical Approach, OCEANS 2006, vol., no., pp.1-9, 18-21 Sept. 2006. DOI=

http://ieeexplore.iee.. rg/stamp/stamp.jsp?tp=\&arnumber= $\underline{4099182 \& \text { isnumber }=4098825}$ 